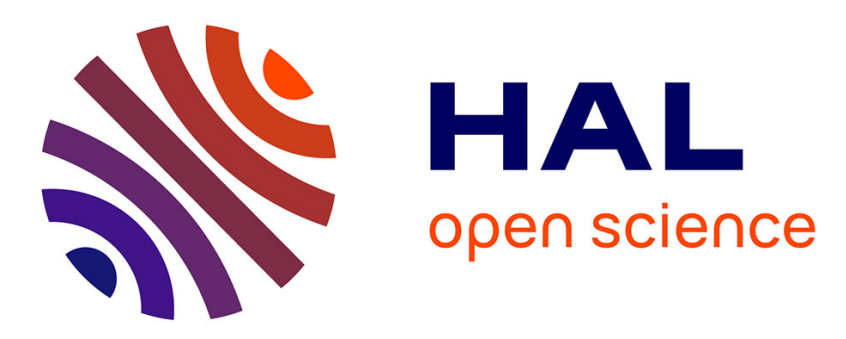

\title{
New developments of a fission chamber for very high radioactivity samples
}

\author{
B. Laurent, J. Taieb, G. Bélier, Paola Marini, P. Morfouace
}

\section{To cite this version:}

B. Laurent, J. Taieb, G. Bélier, Paola Marini, P. Morfouace. New developments of a fission chamber for very high radioactivity samples. Nuclear Instruments and Methods in Physics Research Section A: Accelerators, Spectrometers, Detectors and Associated Equipment, 2021, 990, pp.164966. 10.1016/j.nima.2020.164966 . hal-03368961

\section{HAL Id: hal-03368961 https://hal.science/hal-03368961}

Submitted on 7 Oct 2021

HAL is a multi-disciplinary open access archive for the deposit and dissemination of scientific research documents, whether they are published or not. The documents may come from teaching and research institutions in France or abroad, or from public or private research centers.
L'archive ouverte pluridisciplinaire HAL, est destinée au dépôt et à la diffusion de documents scientifiques de niveau recherche, publiés ou non, émanant des établissements d'enseignement et de recherche français ou étrangers, des laboratoires publics ou privés. 
1

6 Abstract

A new fission chamber was developed and built for prompt-fission-neutron spectra (PFNS) measurements in neutron-induced fission of actinides, and is described in J. Taieb et al., Nucl. Instr. and Meth. A 833 (2016) 1-7. It allowed us to minimize incident- and outgoing-neutron scattering, and optimize both time resolution and alpha to fission fragment discrimination. The fission chamber was validated and used for ${ }^{238} \mathrm{U}$ and ${ }^{252} \mathrm{Cf}$ PFNS measurements. To measure PFNS of samples with a natural alpha activity of the order of $10 \mathrm{MBq}$, further improvements to the detector were done and are presented in this work. Tests of the improved fission chamber lead to a fission fragment detection efficiency of $91 \%$ for a $14.41 \mathrm{MBq}{ }^{240} \mathrm{Pu}$ deposit, and $99 \%$ for a $247 \mathrm{kBq}{ }^{242} \mathrm{Pu}$ deposit. The fission chamber was successfully used for ${ }^{239} \mathrm{Pu}$ PFNS measurements at the Weapons Neutron Research facility of the Los Alamos Neutron Science Center (WNR@LANSCE).

7 Keywords: Fission chamber, high activity samples, efficiency, segmented

8 anode

\section{Introduction}

10

In recent publications [1, 2], a new type of light-weighted fission chamber developed for Prompt-Fission-Neutron Spectra (PFNS) measurements was presented. Actinide mass, time resolution and the amount of structural material were optimized to improve both alpha-fission fragment discrimination and timeof-flight measurements resolution, as well as to reduce the amount of neutrons

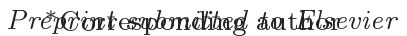

Email address: benoit.laurent@cea.fr (B. Laurent)

${ }^{1}$ Present address: CENBG, CNRS/IN2P3-Université de Bordeaux, Chemin du Solarium B.P. 120, 33175 Gradignan, France

November 30, 2020
} 
scattered from the detector. These chambers were successfully used in PFNS experiments at different facilities: LICORNE at Institut de Physique Nucléaire Orsay (IPNO), Weapon Nuclear Research (WNR) at Los Alamos, 4MV Van de Graaff at Bruyères-le-Chatel. Some results on ${ }^{238} \mathrm{U}(\mathrm{n}, \mathrm{f})$ reactions were published $[2,3]$.

The next step was to use the fission chamber in experiments using high alpha-activity actinides such as ${ }^{239} \mathrm{Pu}$. While the main principle of the detector remains the same (gas type and pressure, gap between anode and cathode), improvements are needed to deal with the high activity of the sample in terms of radioprotection on one hand, and to avoid alpha-signals pile-up, which degrades the alpha-fission fragment discrimination, on the other. By improving this discrimination, cuts on angular and energy distributions of detected fission fragment are reduced and the induced bias on extracted PFNS and others observables (angular distribution, mean neutron energy, number of neutrons emitted per fission...) become negligible. In the following, details of the detector developed for ${ }^{239} \mathrm{Pu}$, but well suited for any other high alpha-activity sample, will be presented.

\section{New design of the chamber}

\subsection{Mechanical aspects}

A schematic view of the fission chamber is shown in Fig. 1. As for the chamber described in [1], the material budget was minimized, to ensure low neutron-scattering rate, thus reducing the neutron background generated during the experiment. Following the same idea, all used materials are almost hydrogen-free. However, because of the high activity of the ${ }^{239} \mathrm{Pu}$ deposits, handling and assembling the targets in the chamber had to be performed in gloves box. Therefore, the external case of the ${ }^{239} \mathrm{Pu}$ detector had to be redesigned to take into account this specificity. Moreover, the dimensions of the detector were increased so that structural material does not intercept the neutron flight path. 
43 When in use, the fission chamber is filled with flowing tetrafluoromethane

$44 \quad\left(\mathrm{CF}_{4}\right)$ gas at a fixed absolute pressure typically 100 mbar above atmospheric 45 pressure.

46 The external structure is a $1 \mathrm{~mm}$-thick aluminium parallelepiped soldered on

47 a $4 \mathrm{~mm}$-thick aluminium flange. The structure supports glued titanium foils on

48 each side: $100 \mu m$ thick on rectangular sides, $50 \mu m$ thick on entrance and exit

49 sides. Titanium foils are glued from the inner side, to stand the gas pressure

so inside the chamber. The soldered flange is screwed on the flange glued to the

51 PCB. An o-ring ensures the gas tightness between the cover and the PCB.

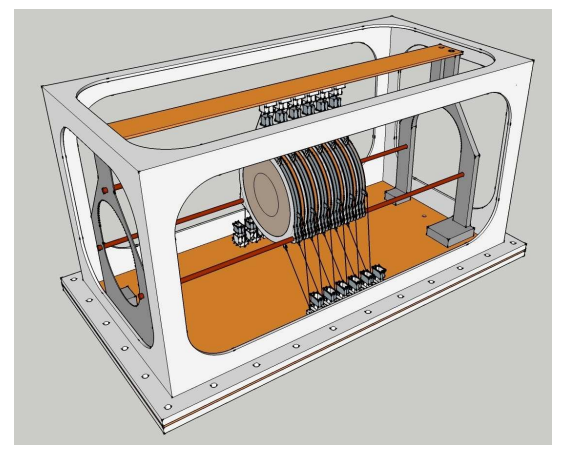

Figure 1: Scheme of the assembled fission chamber (titanium foils are not showned).

52 All the electronic connections and mechanical support were placed on the ${ }_{53}$ bottom side of the chamber. This side consists in a printed circuit board (PCB)

54 supporting all front-end electronics components. The anodes are connected on

55 the internal side, while pre-amplifiers are connected on the external one. The

56 PCB also integrates high voltage connections for the drift field between anodes

57 and cathodes, low voltage connections for pre-amplifiers and a readout connector

58 for each anode. A $3 \mathrm{~mm}$-thick aluminium flange is glued on the external face

59 of the PCB to fix the cover. Aluminium structures fixed on the internal face of

60 the PCB supports three rods made of Torlonß [4] to position the electrodes. 
${ }_{63}$ The sample thickness is a compromise between the amount of matter (and thus

64 the fission rate) and the energy loss of the emitted fission fragments in the deposit. The lower the loss, the better the alpha-fission fragment discrimination.

The Plutonium material was electroplated on a $25 \mu \mathrm{m}$-thick aluminium back-

67 ing (see Fig. 2), glued on the cathode frame. The diameter of the deposit is

$6833 \mathrm{~mm}$ with a thickness of $250 \mu \mathrm{g} / \mathrm{cm}^{2}$. Each deposit contains $\approx 2.1 \mathrm{mg}(5 \mathrm{MBq})$

69 of ${ }^{239} \mathrm{Pu}$ for a total of $47 \mathrm{mg}$ housed in the chamber. The ground connexion of 7o the cathode to the chamber is ensure by a glued cable.

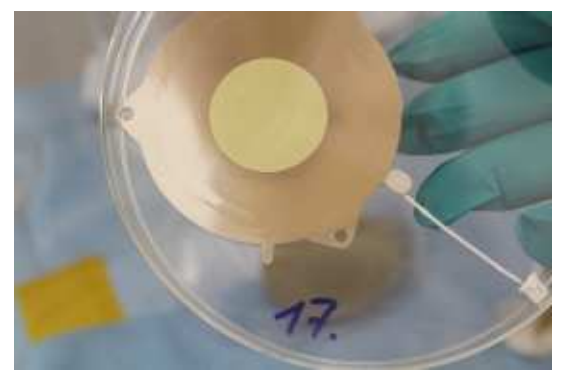

Figure 2: ${ }^{239} \mathrm{Pu}$ sample electroplated on the cathode frame, with glued cable for the ground connexion in the detector.

\subsection{Fission chamber assembly}

The mounting of the $\mathrm{Pu}$ deposits inside the fission chamber was performed at JRC-Geel in a dedicated gloves box. The PCB with internal supports was positioned vertically and then cathodes, spacers and anodes were threaded on the insulating rods (see picture 3(a)). Each anode collects the signal from two deposits (cathodes): one on each side. So there are 11 electronics channels corresponding to the 22 deposits.

Once the 22 cathodes and the 11 anodes are installed, the chamber is closed by the cover (picture 3(b), right), and it can be removed from the gloves box after contamination clean-up. 


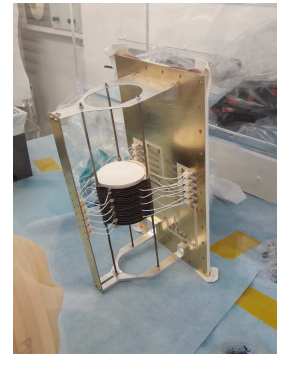

(a)

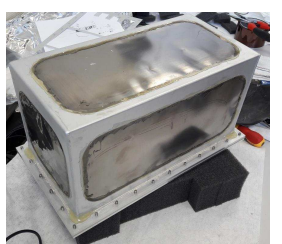

(b)

Figure 3: Pictures of the fission chamber assembly in a gloves box. The stack of anodes and cathodes (with ${ }^{239} \mathrm{Pu}$ ) is mounted (a) before closing the detector with the cover (b).

\section{3. Dedicated segmented anodes}

82 3.1. Anode principle

One anode of the fission chamber deals with $10 \mathrm{MBq}$ alpha activity com-

${ }_{84}$ pared to a standard fission rate during experiments of around $15 \mathrm{f} / \mathrm{s}$. In order to

85 improve alpha-fission fragment discrimination, a dedicated anode was designed.

s6 The goal is to reduce the impact of alpha pile-up on the detection of fission

87 fragments and enhance their discrimination. As the alpha-fission fragment dis-

${ }_{88}$ crimination is based on the different charge-signal amplitudes induced by alpha

89 particles and fission fragments, the worst scenario occurs when an alpha is emit-

9o ted parallel to the cathode. Indeed in this case its deposited charge is close to

91 the one deposited by a fission fragment emitted perpendicularly to the cath-

92 ode. The alpha-particle track length can be longer than the radius of the anode

93 (37 mm), while the track length of the fission fragment will be only the gap

${ }_{94}$ between the anode and the cathode $(2.5 \mathrm{~mm})$, as shown in Fig. 4(a). The anode

95 was therefore segmented in two concentric regions and the collected charge was

96 read only from the central zone. This allowed us to reduce the amount of alpha

97 signals with amplitudes comparable with the ones from fission fragments. The

${ }_{98}$ external part of the anode is isolated from the central part but kept at the same 
99 electrical potential, so not to distort the electric field in the collection region.

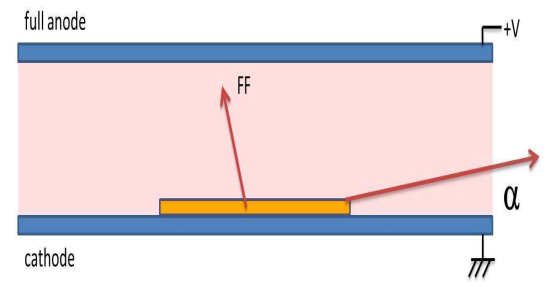

(a)

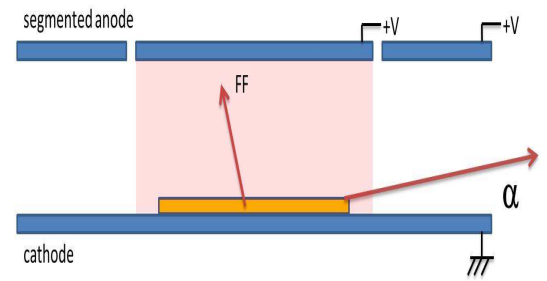

(b)

Figure 4: Principle of the segmented anode. The red zone corresponds to the charge collection zone. In case of a full anode, alphas and alphas pile-up can have greater amplitudes than fission fragments (FF) ones (a). With the segmented anode, this amplitude is reduced (b).

Moreover, a dedicated modern front-end electronics with very short shaping times $(\approx 10 \mathrm{~ns})$ also reduces the signals pile-up and improve the time resolution of the setup.

This peculiar anode design, suitable for high-activity samples fission chamber, have been patented [5].

\subsection{Validation tests with high alpha-activity ${ }^{240,242} \mathrm{Pu}$ deposits}

This new anode concept was tested with high alpha-activity deposits of ${ }^{240,242} \mathrm{Pu}$. These two isotopes are characterized by a very high alpha-activity compared to their spontaneous fission decay rate. They are then good candidates to test offline the limits of alpha-fission fragment discrimination. A 


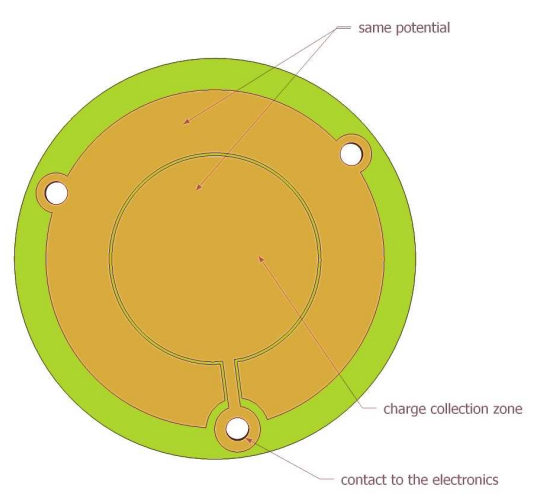

Figure 5: Segmented anode scheme.

14.41 MBq- $\alpha{ }^{240} \mathrm{Pu}$ and $0.247 \mathrm{MBq}-\alpha{ }^{242} \mathrm{Pu}$ deposits were mounted alternatively in the chamber, equipped with plain and then segmented anodes to quantify the improvements. The other characteristics of the chamber were kept constant (gas pressure, gap between anode and cathode, electric field, electronics, DAQ system). Fig. 6 and 7 show the pulse-height spectra collected for these deposits when using a plain anode.

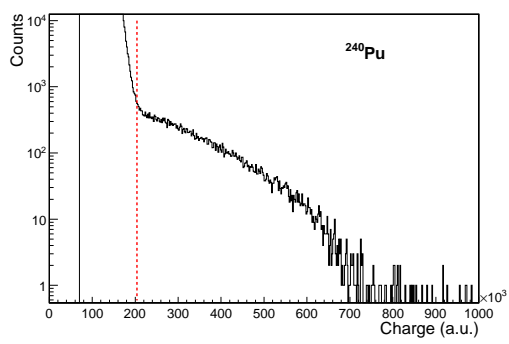

Figure 6: Pulse height spectrum of the ${ }^{240} \mathrm{Pu}$ sample (14.41 MBq), with a fission event detection efficiency of $66 \%$. The red dotted line show the threshold used to determine the number of detected fission fragments.

116 Because of the deposits thickness $\left(0.24 \mathrm{mg} / \mathrm{cm}^{2}\right)$, some of the fission frag17 ments are stopped in the deposit. Monte-Carlo simulations [6] showed that 


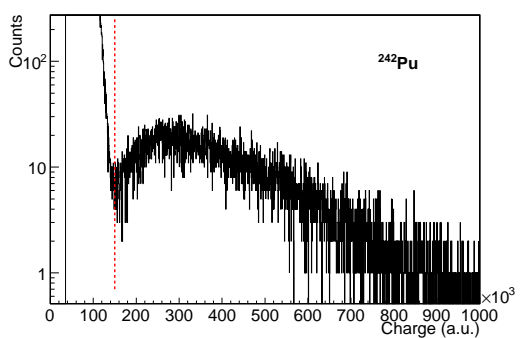

Figure 7: Pulse height spectrum of the ${ }^{242} \mathrm{Pu}$ sample (0.247 MBq), with a fission event detection efficiency of $97 \%$. The red dotted line show the threshold used to determine the number of detected fission fragments.

fission fragments have an efficiency of $94 \%$ to exit the deposits with enough energy to be detected in the gas afterwards. This loss has to be taken into account in the fission detection efficiency calculation.

To improve the alpha-fission fragment discrimination, the time signal of the anode was integrated over two gates and a pulse-shape discrimination (PSD) technique was used. The first gate is centered on the rising part of the signal, while the second one is centered on the maximum amplitude. By plotting the correlation of these two charge signals (Fig. 8), the alpha-fission fragment separation can be improved from $66 \%$ to $91 \%$ for the $14.4 \mathrm{MBq}{ }^{240} \mathrm{Pu}$ sample.

Table 1 summarizes the measured efficiencies for the ${ }^{240,242} \mathrm{Pu}$ samples, in different configurations: plain anode, segmented anode, segmented anode and PSD analysis. These results show that using the segmented anode and a PSD analysis, an efficiency as high as $91 \%$ can be obtained. For the lower activity ${ }^{242} \mathrm{Pu}$ sample (0.247 MBq), the standard fission chamber provides a very good alpha-fission fragment discrimination and the improvements of the detector has less impact.

These tests validate the new concept of anode developed for high alphaactivity actinides used in the fission chamber and new experimental measurements of ${ }^{239} \mathrm{Pu}(\mathrm{n}, \mathrm{f})$ PFNS were performed at WNR@LANSC (see section 5). 
Table 1: Informations concerning ${ }^{240,242} \mathrm{Pu}$ tests, with the values of expected and measured fission rates for each isotope. SF stands for Spontaneous Fission.

\begin{tabular}{|c||c|c|}
\hline Actinide & ${ }^{240} \mathbf{P u}$ & ${ }^{242} \mathbf{P u}$ \\
\hline Alpha activity (MBq) & 14.41 & 0.247 \\
\hline SF branching ratio & $5.7 \mathrm{E}-8$ & $5.5 \mathrm{E}-6$ \\
\hline SF rate (Hz) & 0.82 & 1.36 \\
\hline Fragment emission efficiency & 0.94 & 0.94 \\
\hline Expected fission rate (Hz) & 0.77 & 1.28 \\
\hline \hline \multicolumn{3}{|c|}{ Plain anode } \\
\hline Measured fission rate (Hz) & 0.51 & 1.24 \\
\hline FF efficiency (\%) & $\mathbf{6 6}$ & $\mathbf{9 7}$ \\
\hline \hline \multicolumn{3}{|c|}{ Segmented anode } \\
\hline Measured fission rate (Hz) & 0.60 & 1.25 \\
\hline FF efficiency (\%) & $\mathbf{7 8}$ & $\mathbf{9 8}$ \\
\hline \hline \multicolumn{3}{|c}{ Segmented anode + PSD } \\
\hline Measured fission rate (Hz) & 0.70 & 1.27 \\
\hline FF efficiency (\%) & $\mathbf{9 1}$ & $\mathbf{9 9}$ \\
\hline
\end{tabular}




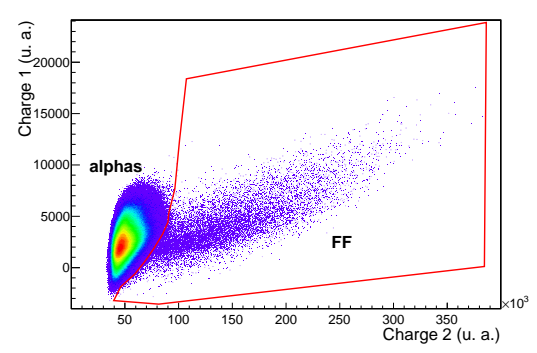

Figure 8: Example of a 2D histrogram showing the alpha-fission fragment discrimination for the ${ }^{240} \mathrm{Pu}$ sample. This histogram is obtained by plotting one charge integration versus the second one (see text for details).

\section{Simulations of the neutrons scattering}

The NPTool framework $[7,8]$ using the Geant4 simulation package was used to quantify the neutron scattering generated by the structural material of the fission chamber. In this work, we used the high precision transport model G4NeutronHP using the default G4NDL nuclear data. During experiments, the fission chamber is placed at the center of an array of 54 liquid-scintillator cells (Chi-Nu cells) as it can be seen in Fig. 9. Both incoming and outgoing neutrons may scatter with the air or with the detector materials, inducing neutron background in the cells and a distortion of the neutron energy spectra. The detailed geometry and material of the fission chamber was carefully included in the simulation (anode, cathode, Ti walls, $\mathrm{Al}$ frame, $\mathrm{PCB} .$. ) In order to quantify the effect of the fission chamber, two different simulations have been performed: one only with air and the second one with the fission chamber included (see Fig. 9). Fig. 10 shows the fraction of incoming neutrons scattered either by the air or by the fission chamber. The blue points correspond to the first simulation only with air while the open red circles show the effect of the added fission chamber. One can see that the fission chamber materials add a negligible background to the prompt-fission neutrons. Moreover, these prompt neutrons of interest are detected in coincidence with a fission, then all scattered neutrons outside the 

reduced by the coincidence gate.

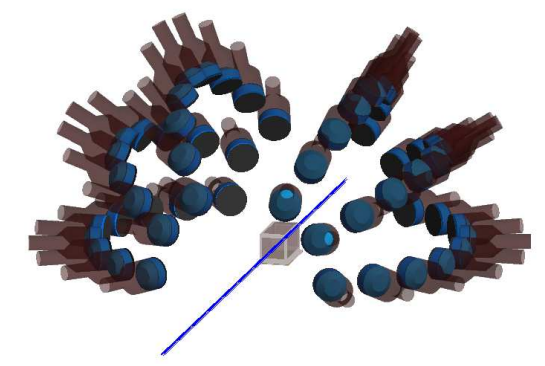

Figure 9: GEANT4 geometry of the fission chamber used in the NPTool simulations. 54 cells surrounding the fission chamber are reproduced. The incoming neutron beam is represented by the blue trajectories.

158

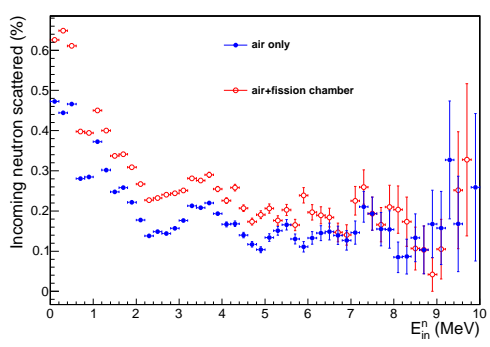

Figure 10: Normalized incoming neutrons scattered on air (blue full circles) and air + fission chamber (red open circles). A maximum of $0.6 \%$ at very low energy of the incoming neutrons are scattered and detected in a Chi-Nu cell, and the effect of the fission chamber is negligible.

\section{Experimental uses}

160

161

${ }^{239} \mathrm{Pu}$ Prompt-Fission-Neutron Spectra (PFNS) were measured placing the fission chamber, containing $47 \mathrm{mg}$ of actinide in the WNR neutron beam to 
induce fission of the $\mathrm{Pu}$ deposits. The detector was surrounded by the 54 liquid scintillator cells of the Chi-Nu array [9]. The incoming-neutron energy is determined by time-of-flight measurement between the beam pulsation and the event detected in the chamber. Neutrons and gammas emitted by neutron-induced fission reaction were detected and identified in the scintillator cells, and neutrons energies measured via time-of-flight technique. Results of this experiment were presented in [10]. As an example, Fig. 11 shows the pulse-height spectra of ${ }^{239} \mathrm{Pu}$ fission chamber with and without beam. The fission detection efficiency was estimated to be close to $95 \%$ for $10 \mathrm{MBq}$ alpha activity per channel, thanks to some improvements on the design of the front-end electronics boards.

This good fission event identification probability, combined the very low neutron detection threshold $(\approx 200 \mathrm{keV})$ and the granularity of the Chi-Nu detector, allowing angular emission corrections, as well as the high recorded statistics, lead to very precise PFNS measurements. Moreover, thanks to these characteristics, the number of neutrons emitted per fission $(\bar{\nu})$ as a function of incoming energy was extracted from the measurement with an unprecedented accuracy [11].

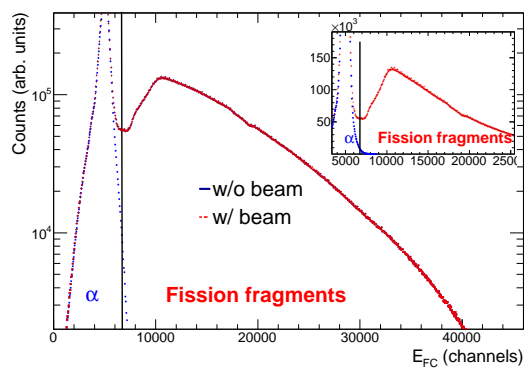

Figure 11: Pulse height spectra of ${ }^{239} \mathrm{Pu}$ fission chamber obtained with (red) and without (blue) beam (extracted from [10]). 


\section{Conclusions}

The fission chamber developed for PFNS measurements was improved to be used with high alpha-activity samples. The housing was modified to allow the assembly of the detector in a gloves box. For fission detection, a new concept of segmented anode was developed. Thanks to the use of these segmented anodes and modern front-end electronics, a fission detection efficiency of $95 \%$ was reached for alpha activities as high as $10 \mathrm{MBq}$, to be compared to a fission rate of $15 \mathrm{f} / \mathrm{s}$.

Thanks to its very high alpha-fission fragment discrimination capability, this fission chamber can be further used in fission cross-section as well as PFNS measurements of very active actinides, such as ${ }^{238,240,241,242} \mathrm{Pu}$.

\section{References}

[1] J. Taieb, B. Laurent, G. Bélier, A. Sardet, C. Varignon, Nucl. Instr. and Meth. A 833 (2016) 1-7.

[2] A. Sardet, C. Varignon, B. Laurent, T. Granier, A. Oberstedt, Nucl. Instr. and Meth. A 792 (2015) 74-80.

[3] B. Laurent, P. Marini, G. Bélier, T. Bonnet, A. Chatillon, J. Taieb, D. Etasse, M. Devlin, R. Haight, EPJ Web Conf. 146 (2017) 04014.

[4] Torlon, Solvay specialty polymers, http://www.solvayplastics.com/.

[5] J. Taieb, B. Laurent, Fr. patent 3069700, 2019-02-01.

[6] L. Mathieu, private communication, 2015.

[7] A. Matta, P. Morfouace, N. de Séréville, F. Flavigny, M. Labiche, R. Shearman, Journal of Physics G: Nuclear and Particle Physics 43 (2016) 045113.

[8] NPTool, a ROOT/Geant4 based framework for Nuclear Physics, https://gitlab.in2p3.fr/np/nptool, consulted on November 2020. 
205 [9] R. C. Haight, H. Y. Lee, T. N. Taddeucci, J. M. O'Donnell, B. A. Perdue, 206 N. Fotiades, M. Devlin, J. L. Ullmann, A. Laptev, T. Bredeweg, M. Jandel, 207 R. O. Nelson, S. A. Wender, M. C. White, C. Y. Wu, E. Kwan, A. Chyzh, R. Henderson, J. Gostic, J. Instrum. 7 (2012) C03028.

[10] P. Marini, J. Taieb, B. Laurent, G. Belier, A. Chatillon, D. Etasse, P. Morfouace, M. Devlin, J. A. Gomez, R. C. Haight, K. J. Kelly, J. M. O’Donnell, K. T. Schmitt, Phys. Rev. C 101 (2020) 044614.

[11] P. Marini, J. Taieb, G. Belier, A. Chatillon, D. Etasse, B. Laurent, P. Morfouace, M. Devlin, J. A. Gomez, R. C. Haight, K. J. Kelly, J. M. O’Donnell, submitted to Phys. Rev. Lett. (Nov. 2020). 